\title{
Comets and the Connection to Life
}

\author{
K. J. Meech \& J. M. Bauer \\ Institute for Astronomy, 2680 Woodlawn Dr., Honolulu, HI 96822
}

\begin{abstract}
We present a summary of ground-based work being done to gain an understanding of primitive comet, Centaur and Kuiper belt object compositions. We are seeing a diversity of compositions in outer solar system small bodies with respect to the presence of water and organics which may reflect both primordial differences and evolutionary processes.
\end{abstract}

\section{Introduction}

Ground-based and space-based observations of comets, Centaurs and TransNeptunian or Kuiper belt objects are revealing a fascinating diversity of surface properties and compositions, including evidence for organics. Understanding the presence of organics, their alteration history and inventory is an important step in understanding pre-biotic environments. These studies figure prominently in many current, planned and proposed space missions and investigations, and are especially important in our reconnaissance of the small icy solar system bodies which represent our most primitive reservoir of early solar system material.

There are 4 recent and upcoming missions to comets which are designed to give us an understanding of the fundamental chemical and physical nature of these primordial remnants of the early solar system. These missions are shown in Table 1. The data from these missions will give us detailed observations of the surface and near sub-surface properties of a handful of short period comets; however, understanding the context of these bodies with respect to solar system formation will require a detailed comparison with a statistical sample of small outer solar system bodies which formed at a variety of different nebular locations.

Table 1. Comet Missions

\begin{tabular}{lcccl}
\hline Mission & Target & Launch & Encounter & Goals \\
\hline Deep Space 1 & 19P/Borrelly & $10 / 24 / 98$ & $09 / 22 / 01$ & Flyby imaging \\
Stardust & 81P/Wild 2 & $02 / 07 / 99$ & $01 / 15 / 06$ & Dust return \\
Deep Impact & 9P/Tempel 1 & $01 / 04$ & $07 / 04 / 05$ & Cratering expt \\
Rosetta (ESA) & 46P/Wirtanen & $01 / 13 / 03$ & $11 / 11-10 / 13$ & Rendezvous \\
\hline \hline
\end{tabular}




\subsection{Formation and Evolution}

Comets are the icy remnants from the process of solar system formation and depending on the extent of their subsequent evolution, may provide keys to the chemical and physical conditions in the nebula, and the possible preservation of interstellar and pre-biotic material. Comets formed in the region extending from the giant planets out into the Kuiper belt, probably from shock heated gases recondensed onto interstellar grain cores which may have partially survived infall into the nebula. Throughout much of the nebular region in which the comets formed, the equilibrium temperatures would have been below $100 \mathrm{~K}$, allowing the water to condense in an amorphous form, trapping other more volatile gases. This process is extremely temperature sensitive, thus the abundance and composition of super-volatiles provides a sensitive cosmic thermometer of nebular conditions.

\section{ACTIVITY LEVELS FOR DYNAMICAL CLASSES}

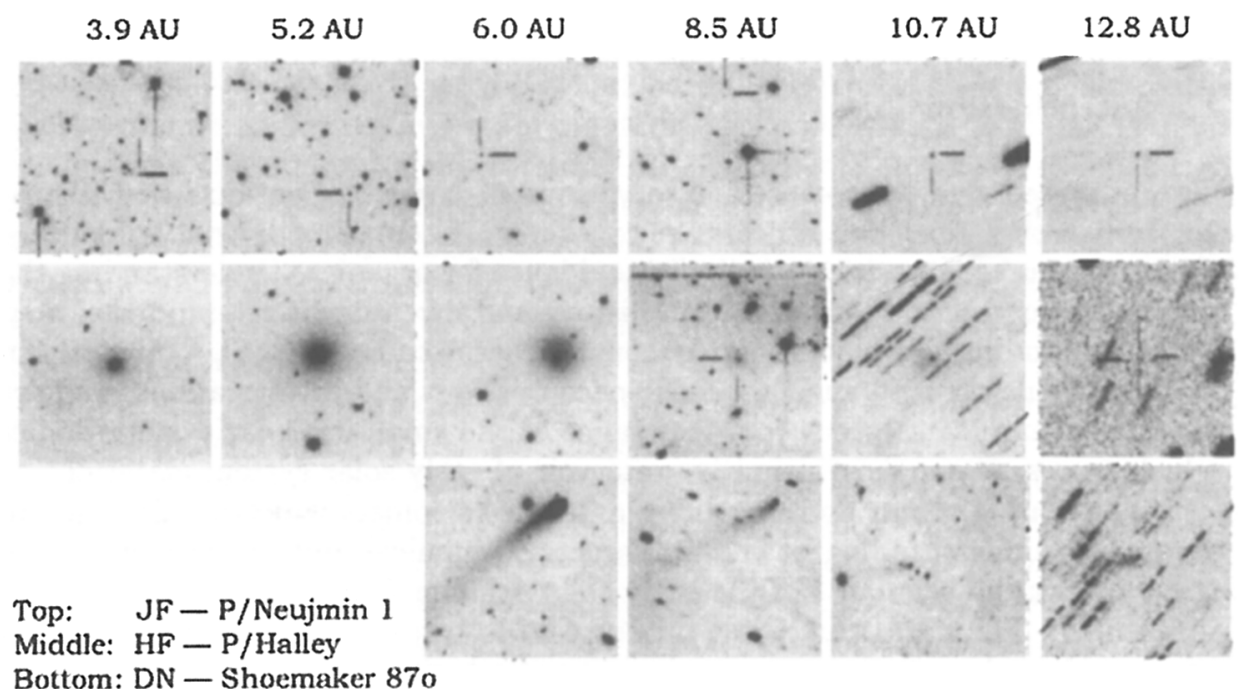

Figure 1. Differences in light curve behavior and level of activity as a function of dynamical class.

Comets which formed in the giant planet region were for the most part ejected into the distant Oort cloud through giant planet perturbations. These comets, having formed at higher temperatures, would have trapped fewer volatiles than their counterparts forming further out. Dynamically the Oort comets evolve to become the dynamically new (DN) comets making their first passage through the inner solar system, and the long period (LP) comets, which contribute a small amount to the Halley type (HF) short-period (SP) comet population. The objects in residence in the Kuiper Belt beyond Pluto probably formed largely in-situ at much lower temperatures where trapping volatile species was easier. Centaurs are the objects evolving dynamically inwards from the Kuiper belt into SP comet orbits. 
However, since the time of formation, all small bodies have undergone evolutionary processing from cosmic ray bombardment, collision and heating, especially those having spent time within the inner solar system. Thus, the surfaces and escaped volatiles of different dynamical populations may not reflect differences in primordial chemistry. Figure 1 illustrates the differences in activity levels among comets of different dynamical classes. These comets are characterized by dramatically different activity levels and appearance - probably a combination of evolution and differences in intrinsic composition (Meech 1999).

\subsection{Observing Techniques}

The standard techniques for getting information about the composition of comets involves spectroscopy of bright comets, optical photometry, and infrared and radio detection of parent volatile species. The difficulty with spectroscopic observations of bright accessible comets is that the volatiles are sublimated from the nucleus and shortly after entering the coma can experience photolysis, and photodissociation to daughter species. Models are used to infer the abundance of various parent species from the observed band strengths. With recently observed bright comets such as C/1995 O1 Hale Bopp, C/1996 B2 Hyakutake, and $\mathrm{C} / 1999 \mathrm{H} 1 \mathrm{Lee}$, it was possible to make direct observations of the parent molecules in the infrared and radio. A summary of the range of comet compositions is shown in Table 2, and shows similarities to the composition of gases in molecular clouds.

Table 2. Relative Abundances of Cometary Volatiles

\begin{tabular}{llll}
\hline Molecule & Abundance & Molecule & Abundance \\
\hline $\mathrm{CO}$ & $1-20$ & $\mathrm{HNCO}$ & 0.1 \\
$\mathrm{CS}$ & 0.2 & $\mathrm{C}_{2} \mathrm{H}_{2}$ & $\sim 0.1$ \\
$\mathrm{SO}$ & $\sim 0.5$ & $\mathrm{NH}_{3}$ & 0.6 \\
$\mathrm{~S}_{2}$ & 0.005 & $\mathrm{H}_{2} \mathrm{CS}$ & $\sim 0.02$ \\
$\mathrm{H}_{2} \mathrm{O}$ & 100 & $\mathrm{HCOOH}$ & $\sim 0.05$ \\
$\mathrm{CO}_{2}$ & $3-20$ & $\mathrm{CH}_{4}$ & $\sim 0.6$ \\
$\mathrm{HCN}$ & 0.2 & $\mathrm{HC}_{3} \mathrm{~N}$ & 0.03 \\
$\mathrm{HNC}$ & 0.04 & $\mathrm{CH}_{3} \mathrm{OH}$ & $1-7$ \\
$\mathrm{H}_{2} \mathrm{~S}$ & $0.2-1.5$ & $\mathrm{HN}_{2} \mathrm{CHO}$ & $\sim 0.01$ \\
$\mathrm{OCS}$ & 0.5 & $\mathrm{CH}_{3} \mathrm{CN}$ & 0.02 \\
$\mathrm{SO}_{2}$ & $\sim 0.1$ & $\mathrm{HCOOCH}_{3}$ & $\sim 0.05$ \\
$\mathrm{H}_{2} \mathrm{CO}$ & $0.1-1$ & $\mathrm{C}_{2} \mathrm{H}_{6}$ & $\sim 0.3$ \\
\hline \hline
\end{tabular}

Note: Data are from Irvine et al. (2000) and show abundances relative to water.

One of the surprising things learned from these bright comets is that it is very likely that chemistry plays a very important role in the inner coma, and thus these types of observations may not in fact reflect the primordial composition in the cometary interior and may not be entirely useful indicators of the preservation of interstellar material. Direct spectral and photometric observa- 
tions of small body surfaces avoid the problem of coma models and chemistry in the inner coma; however the surfaces of the small bodies are likely to be different from the interiors because of evolution.

\section{Spectral Diversity of Small Bodies}

As discoveries of Kuiper belt objects (KBOs) and Centaurs became routine, attention was turned to their characterization. Tegler \& Romanishin (1998) suggested from their observations of the colors of KBOs that the objects fell into two distinct compositional classes while other groups did not see the bimodal distribution in their data. There is now a large statistical ensemble of Kuiper belt (Hainaut \& Delsanti 2002; Delsanti et al. 2001) and Centaur colors (see Fig. 2) which show a continuous broad distribution of colors (Bauer, in prep.). The colors of the KBOs and Centaurs are broader than those of comet nuclei, suggesting a more diverse suite of surface properties (Meech, Hainaut, \& Marsden 2003). It is too early to determine if these differences are essentially primordial, evolutionary or a combination of both.

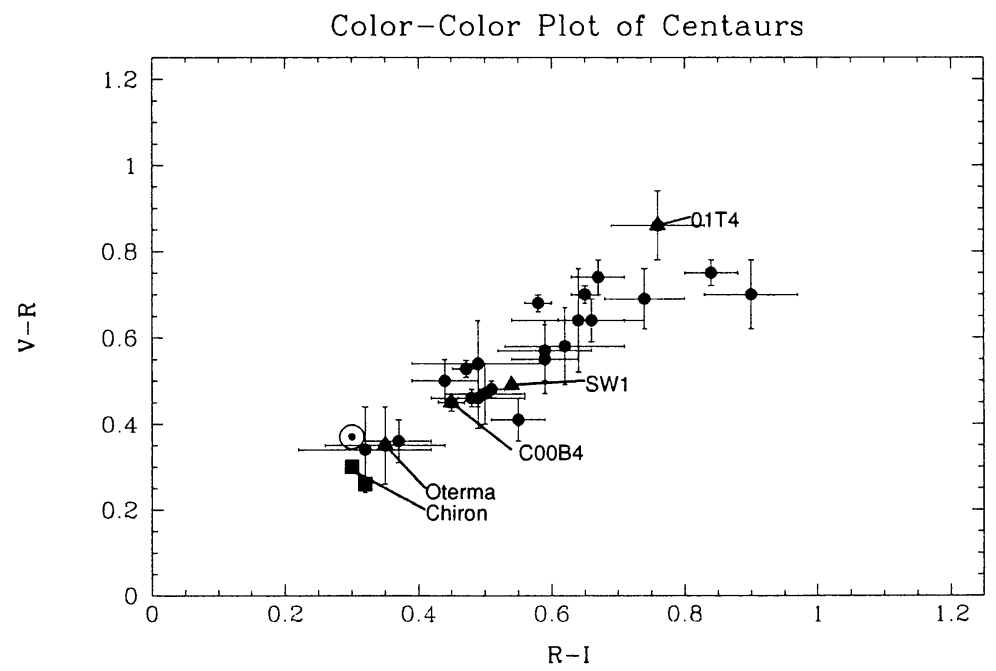

Figure 2. The V-R and R-I colors of 24 Centaurs with 1- $\sigma$ errors. The data points with labels indicate Centaurs with reported activity. The solar colors are indicated by $\odot$.

Table 3 summarizes the properties of the Centaurs and KBOs with spectral features. The spectra are modelled with mixtures of water-ice, organic material and amorphous carbon (to lower the albedo). The other objects for which observations exist show featureless spectra, but with a wide range of slopes. Cruikshank et al. (2002) have suggested that D-type spectral slopes, similar to the red surfaces seen in distant asteroids, can be matched by pyroxenes, but spectra which are much redder than this require the presence of organic materials. Apparently there is an abundance of organic material in these small outer solar system cometary bodies. 
Table 3. Small Body Spectral Features

\begin{tabular}{lcl}
\hline Object & Type & Features \\
\hline $1997 \mathrm{CU}_{26}$ & Centaur & $3 \% \mathrm{H}_{2} \mathrm{O}$, Murchison \\
2060 Chiron & Centaur & some $\mathrm{H}_{2} \mathrm{O}$ \\
5145 Pholus & Centaur & $\mathrm{H}_{2} \mathrm{O}, \mathrm{NH}_{3}$, Titan Tholin \\
8405 Asbolus & Centaur & $\mathrm{H}_{2} \mathrm{O}$ \\
$1999 \mathrm{UG}_{5}$ & Centaur & $\mathrm{H}_{2} \mathrm{O}$ ? organics? \\
$2001 \mathrm{PT}_{13}$ & Centaur & $\mathrm{H}_{2} \mathrm{O}$, Titan tholins, carbon \\
$1996 \mathrm{TO}_{66}$ & $\mathrm{KBO}$ & $\mathrm{H}_{2} \mathrm{O}$ \\
$1993 \mathrm{SC}$ & $\mathrm{KBO}$ & $\mathrm{H} y d r o c a r b o n s ?$ \\
$1996 \mathrm{TQ}_{66}$ & $\mathrm{KBO}$ & $1.9 \mu$ m feature \\
$1996 \mathrm{TS}_{66}$ & $\mathrm{KBO}$ & $1.9 \mu$ m feature \\
7 others & $\mathrm{KBO}$ & Featureless \\
\hline \hline
\end{tabular}

\subsection{Centaurs with Water Features}

Water ice has been seen in the spectra of 6 small outer solar system bodies. Figure 3 shows a comparison of the near-infrared spectra of these objects showing the water absorption bands near 1.5 and $2.0 \mu \mathrm{m}$. Some of these objects appear to exhibit variations in surface properties both as a function of rotational phase, and as a function of time.

Surface Changes $\mathrm{KBO} 1996 \mathrm{TO}_{66}$ exhibited an unusual change in the shape of its rotational light curve between 1997 and 1998 which showed that either the period had changed significantly, or that the surface albedo had changed significantly on parts of the object (Hainaut et al. 2000). Models of activity on the surface could account for such a change in brightness if portions of a dark radiation damaged surface had been removed, say through cometary activity, exposing a higher albedo region. This is also consistent with surface colors which varied with rotation in data taken in 1999.

Possible surface variations were also seen on Centaur 8405 Asbolus which has a rotation period of 8.9351 hours. Kern et al. (2000) reported significant changes in the absorption features in spectra taken over a time period of 1.7 hours - where $\mathrm{H}_{2} \mathrm{O}$-ice features were seen at one epoch and not the other. Data taken on a later date, showed no spectral features at all during a full rotation period (Romon-Martin et al. 2001), again suggesting that something has altered the surface materials, with a likely candidate being activity.

Unique Surfaces The spectrum of Centaur $1995 \mathrm{UG}_{5}$ shows a unique surface. There are possible absorptions corresponding to the 1.5 and $2.0 \mu \mathrm{m}$ bands of water, but these absorption features are only present at the peak brightness in the optical rotational lightcurve. In addition the slope of the spectrum is very red at peak brightness, which requires the presence of organic material. The fact that the water ice band is on a redder and brighter region of the surface is somewhat surprising. Reddening and darkening are often associated with older 
irradiated surfaces. Observations as a function of phase angle show a very steep behavior which may be indicative of a very porous surface.

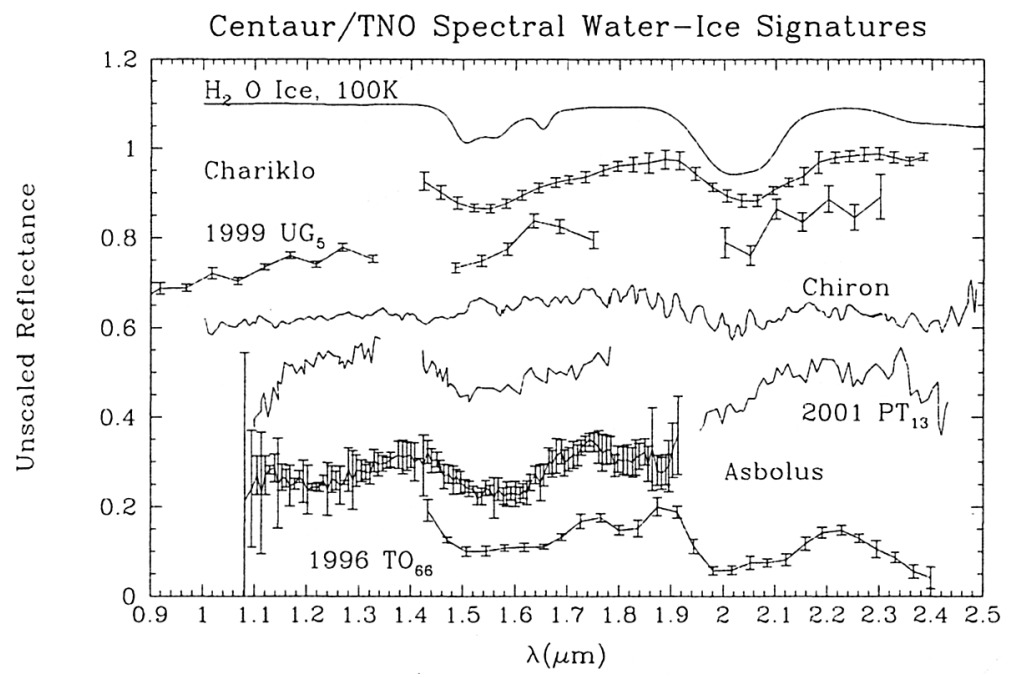

Figure 3. Comparison of the near-IR water ice signatures in 6 Centaurs and KBOs, including Chariklo (Brown et al. 1998), $1999 \mathrm{UG}_{5}$ (Bauer et al. 2002a), Chiron (Luu, Jewitt, \& Trujillo 2000), $2001 \mathrm{PT}_{13}$ (Barucci et al. 2002), Asbolus (Kern et al. 2000), and $1996 \mathrm{TO}_{66}$ (Brown, Cruikshank, \& Pendleton 1999). The water ice spectrum is derived from Grundy \& Schmitt (1998), and each of the spectra is arbitrarily scaled to fit on the figure.

This investigation of outer solar system small body compositions is not restricted to cometary bodies, but is being extended to outer solar system satellites, many of which may be captured bodies. A spectrum from 1.2 to $2.5 \mu \mathrm{m}$ of Uranus' small satellite Miranda obtained in June 1999 (Bauer et al. 2002b) reveals strong water ice signatures, in addition to a weak absorption band at $1.65 \mu \mathrm{m}$ that is indicative of crystalline water ice (see Fig. 4). The crystalline water ice implies heating of the surface to temperatures above $142 \mathrm{~K}$. The models show that the surface is dominated by water ice, with a large fraction of carbonaceous or silicate contaminates, and the possible presence of ammonia hydrate (at $2.2 \mu \mathrm{m}$ ). This, and possible features reported on Charon (Dumas et al. 2001), would be the only spectroscopic evidence to date of an ammonia species in the outer solar system beyond Saturn.

\section{Discussion}

Not only has the discovery of the Kuiper belt given us enormous insight into the dynamical evolution of the outer solar system, and the mass of the solar nebula, but as we begin to obtain detailed physical data on the surface compositions of these objects we are seeing an unexpected diversity of surface composition which likely reflects a combination of ongoing processes (activity?, collision, irradiation) as well as differences in primordial chemistry. The outer solar system 


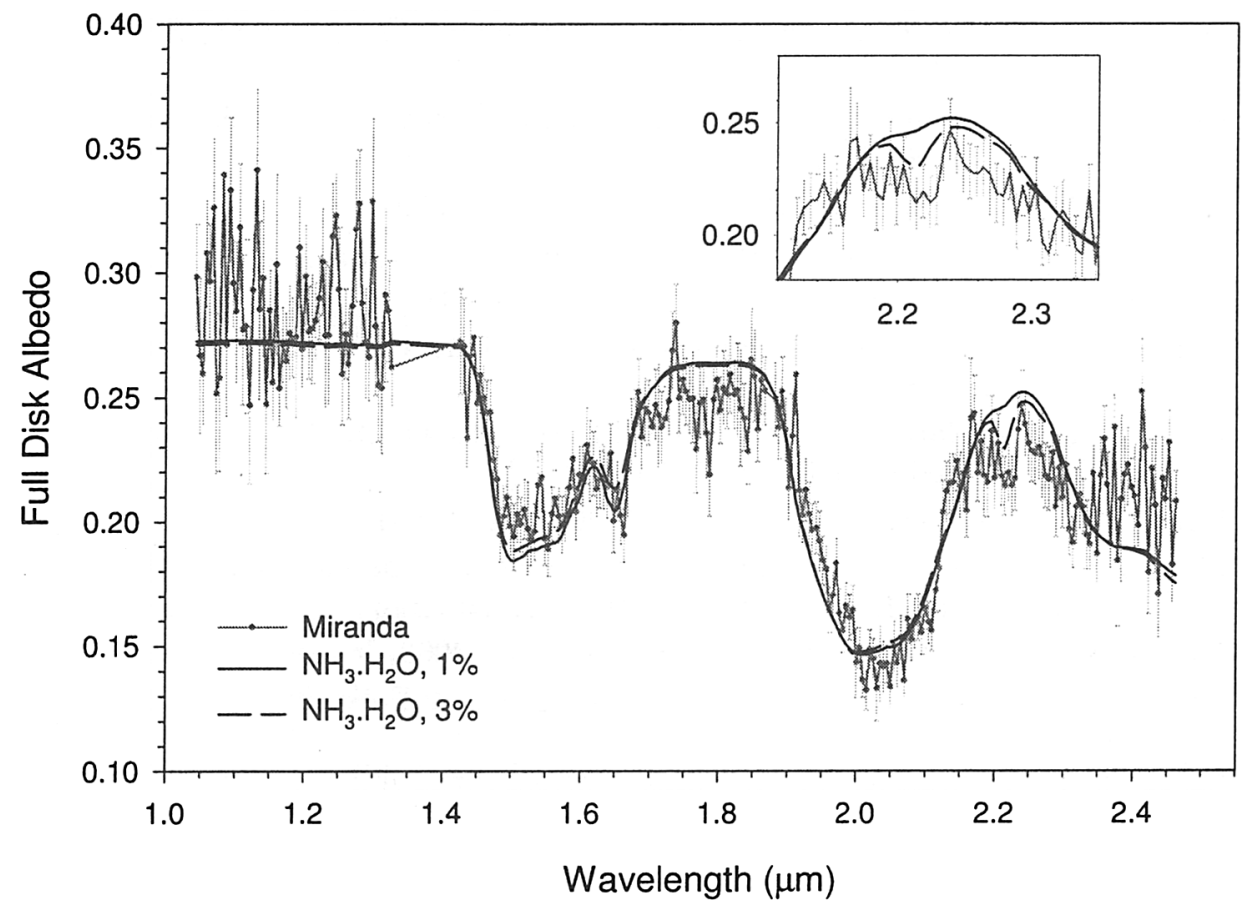

Figure 4. Comparison of a spatially mixed model from Bauer et al. (2002b) incorporating ammonia hydrate ice to the $9 / 00$ Miranda observations. The slight shoulder seen near $2.22 \mu \mathrm{m}$ is stronger in higher concentrations of ammonia hydrate ices.

has abundant organic material, and through perturbations this gets sent inwards to the region of the terrestrial planets, and this has enormous impact on issues related to pre-biotic chemistry and the evolution of life.

Acknowledgments. This research was supported in part by a grant from a grant from NASA (NAG5-4495).

\section{References}

Barucci, M. A., et al. 2002, A\&A, 392, 335

Bauer, J. M., Meech, K. J., Fernández, Y. R., Farnham, T., Roush, T., \& Owen, T. C. 2002a, PASP, in press

Bauer, J. M., et al. 2002b, Icarus, 158, 178

Brown, R. H., Cruikshank, D. P., Pendleton, Y., \& Veeder, G. J. 1998, ApJ, 519, L101

Brown, R. H., Cruikshank, D. P., \& Pendleton, Y. 1999, Science, 280, 1430

Cruikshank, D. P., Dalle Ore, C. M., Roush, T. L., \& Khare, B. N. 2002, BAAS, $34,07.09$ 
Dumas, C., Terrile, R. J., Brown, R. H., Schneider, G., \& Smith, B. A. 2001, AJ, 121, 1163.

Delsanti, A. C., Boehnhardt, H., Barrera, L., Meech, K. J., Sekiguchi, T., \& Hainaut, O. R. 2001, A\&A, 380, 347

Grundy, W. M., \& Schmitt, B. 1998, JGR, 103, 25809

Hainaut, O. R., et al. 2000, A\&A, 356, 1076

Hainaut, O. R., \& Delsanti, A. 2002, A\&A, 389, 641

Irvine, W. M., et al. 2000, in Protostars and Planets IV, ed. V. Mannings, A. P. Boss \& S. S. Russell, 1159

Kern, S. D., McCarthy, D. W., Buie, M. W., Brown, R. H., Campins, H., \& Rieke, M. 2000, ApJ, 542, L155

Luu, J. X., Jewitt, D. C., \& Trujillo, C. 2000, ApJ, 531, L151

Meech, K. J. 1999, in Evolution and Source Regions of Asteroids and Comets, ed. J. Svoren, E. M. Pittich, \& H. Rickman, 195

Meech, K. J., Hainaut, O. R., \& Marsden, B. G. 2003, Icarus, in press

Romon-Martin, J., Barucci, M. A., de Bergh, C., \& Peixinho, N. 2001, BAAS, $33,8.05$

Tegler, S. C., \& Romanishin, W. 1998, Nature, 392, 49 